\title{
Rat Strains
}

National Cancer Institute

\section{Source}

National Cancer Institute. Rat Strains. NCI Thesaurus. Code C14417.

Breeds of rats utilized for biological research. 